\title{
Community-Based Health Education Programs Designed to Improve Clinical Measures Are Unlikely to Reduce Short-Term Costs or Utilization Without Additional Features Targeting These Outcomes
}

\author{
Joe Burton, MS, Barry Eggleston, MS, Jeffrey Brenner, MD, ${ }^{3,4}$ Aaron Truchil, MS, \\ Brittany A. Zulkiewicz, BS, and Megan A. Lewis, PhD²
}

\begin{abstract}
Stakeholders often expect programs for persons with chronic conditions to "bend the cost curve." This study assessed whether a diabetes self-management education (DSME) program offered as part of a multicomponent initiative could affect emergency department (ED) visits, hospital stays, and the associated costs for an underserved population in addition to the clinical indicators that DSME programs attempt to improve. The program was implemented in Camden, New Jersey, by the Camden Coalition of Healthcare Providers to address disparities in diabetes care. Data used are from medical records and from patient-level information about hospital services from Camden's hospitals. Using multivariate regression models to control for individual characteristics, changes in utilization over time and changes relative to 2 comparison groups were assessed. No reductions in ED visits, inpatient stays, or costs for participants were found over time or relative to the comparison groups. High utilization rates and costs for diabetes are associated with longer term disease progression and its sequelae; thus, DSME or peer support may not affect these in the near term. Some clinical indicators improved among participants, and these might lead to fewer costly adverse health events in the future. DSME deployed at the community level, without explicit segmentation and targeting of high health care utilizers or without components designed to affect costs and utilization, should not be expected to reduce shortterm medical needs for participating individuals or care-seeking behaviors such that utilization is reduced. Stakeholders must include financial outcomes in a program's design if those outcomes are to improve.
\end{abstract}

\section{Introduction}

$\mathbf{N}$ EARLY 29 MILLION AMERICANS (or about 9\% of the US population) have diabetes. ${ }^{1,2}$ Among people with diabetes, racial/ethnic minorities are disproportionally affected by the disease. Compared with non-Hispanic white adults, the risk of diagnosed diabetes in 2010 was $18 \%$ higher among Asian Americans, 66\% higher among Hispanics, and 77\% higher among non-Hispanic blacks. ${ }^{2}$ Data from the 2009 Indian Health Service's (IHS) National Patient Information
Reporting System show that $\sim 14 \%$ of American Indians and Alaska Natives aged 20 years or older who received care from the IHS had diabetes. ${ }^{2}$ In addition, gaps exist between the percentage of non-Hispanic whites who receive all 3 recommended diabetes clinical services (hemoglobin A1c testing, eye examination, and foot examination; 42.4\%) and the proportion of Hispanics who do (33.8\%). In 2005, there were differences between the percentages of blacks and whites who received these services $(37 \%$ and $41 \%$, respectively) as well; however, they were not statistically

\footnotetext{
${ }^{1}$ RTI International, Waltham, Massachusetts.

${ }^{2}$ RTI International, Research Triangle Park, North Carolina.

${ }^{3}$ Camden Coalition of Healthcare Providers, Camden, New Jersey.

${ }^{4}$ Cooper University Hospital, Camden, New Jersey.
}

(C) Burton et al, 2016; Published by Mary Ann Liebert, Inc. This Open Access article is distributed under the terms of the Creative Commons Attribution Noncommercial License (http://creativecommons.org/licenses/by-nc/4.0/) which permits any noncommercial use, distribution, and reproduction in any medium, provided the original author(s) and the source are credited. 
significant. ${ }^{3}$ These statistics also point to the large financial burden that diabetes places on health systems, communities, and patients managing diabetes.

The prevalence of diabetes in the United States is expected to double over the next 25 years, bringing about related increases in service needs and costs. ${ }^{4}$ The largest increases in prevalence will be among groups currently experiencing the largest diabetes burden, such as blacks. ${ }^{5}$ These increases will require large-scale implementation of evidence-based approaches across the United States to help patients, communities, and health systems manage diabetes, stem disease progression, and potentially slow the increase of health care expenditures caused by diabetes.

Since 2007, the Camden, New Jersey Coalition of Healthcare Providers (the Coalition) has coordinated a citywide project to provide care management and transitional primary care for city residents who are the highest users of Camden's emergency departments (EDs) and hospitals in an effort to improve quality and reduce expensive hospital utilization among this high-utilizing group. The Coalition successfully piloted this "hot-spotting" technique and was among the first organizations to systematically identify and intervene with high users using multidisciplinary, community-based teams as a way to decrease utilization. ${ }^{6,7}$ In 2009, the Coalition extended this work to include a specific focus on diabetes with a 5-year, multilevel, multicomponent initiative to help address health and health care disparities with respect to type 2 diabetes. The initiative included both a focus on high utilizers, for whom case management was used to help control their diabetes progression, and the broader population of individuals with type 2 diabetes, for whom group-based diabetes selfmanagement programs were developed. ${ }^{8}$

\section{Purpose}

The purpose of this study is to test whether the strategies implemented in Camden through the Coalition's Diabetes Self-Management Education (DSME) program reduced hospital utilization and costs. Some evidence suggests that intervening with high utilizers of health care, as the Coalition has done before, can decrease costs. ${ }^{9-11}$ The Coalition demonstrated some cost savings in a small group of high utilizers who had diabetes, ${ }^{9}$ but this association has not been tested in a group of patients who are receiving communitybased, self-management education. The study team hypothesized that community-based DSME provided to individuals without a case management component would have a small but detectable effect on hospital utilization and costs, and specifically examined whether the program decreased the use of hospital services in the short term. The team also hypothesized that persons who completed the DSME program would have lower rates of ED use and inpatient stays in the following year compared to individuals who participated but did not complete the program.

\section{The Camden DSME program}

Diabetes self-management interventions aim to improve glycemic control, improve other clinical indicators, and prevent acute and chronic complications. The Camden program has 5 community-based diabetes mellitus education sites based on the American Diabetes Association/American
Association of Diabetes Educators guidelines. The program offers an 8-class diabetes education and nutrition curriculum in both English and Spanish. The program also hosts "Ask a Heath Provider," which occurs weekly for 2 hours, and "Diabetes Day," which occurs monthly. The DSME program also incorporated features of Project DULCE, a community-based, culturally appropriate, nurse case management, peer education diabetes care model. ${ }^{12}$ Internal medicine providers refer patients to a nurse coordinator who will provide a 50-minute visit focused on building rapport and teaching diabetes management. The nurse will act as a case manager and help patients to access appropriate resources while also acting as a liaison to providers. Each patient then begins an 8-week course learning an evidencebased DSME curriculum from a peer educator, a Camden resident with diabetes. Participation is defined as attendance at 1 or more of the classes offered. Thirteen cohorts of enrolled patients completed the DSME program developed by the Coalition.

\section{Data}

Two data sets were used for the analyses: (1) data from medical records and (2) data from the Camden Health Database, a citywide data set of hospital encounters. ${ }^{6}$

\section{Data from medical records}

The Coalition constructed a data set from medical and participation records for use in the evaluation of its program. The data included participation and demographic information for approximately 125 participants as well as clinical measures for a subset of those participants. The study team used these data to assess changes in selected clinical measures. The team also used them to assign participants as "high-intensity" or "low-intensity" participants because it was hypothesized that the high-intensity group would be more likely than the low-intensity group to have lower hospital utilization and costs after completion of the program. High intensity indicates that individuals attended 6 or more of the 8 sessions and were considered to have "graduated"; low intensity indicates that they attended at least 1 and up to 5 sessions in the curriculum. The enrollment data set also included demographic information on 84 additional individuals who met the criteria to be part of the DSME program and who were on the recruitment roster but who did not participate. All individuals had been diagnosed with type 2 diabetes.

\section{The Camden Health Database}

The Camden Health Database is an aggregate of 10 years of patient and visit-level information about hospital services and health care cost data from Camden's 3 major health systems. The data set includes information about the use of hospital services (inpatient stays, observation stays, and ED visits) as well as demographic, financial, and clinical variables about those who received services. The file contained discharge abstracts for 132,454 unique individuals with admissions for calendar years from 2002 through 2012 (the latest discharge date was February 24, 2013). Important analytic variables included age, sex, zip code, admission and discharge dates, source of payment (auto insurance, charity, 
commercial, correctional, Medicaid, Medicare, military, self-pay, workers' compensation), charges and receipts, and diagnoses. The participation and medical record data were linked to the Camden Health Database using probabilistic matching techniques to assess hospital utilization and costs over time among participants in the DSME program. The study team also used the data set to construct a comparison group of individuals with characteristics similar to those of the participants. Because the Camden Health Database only includes data for Camden residents visiting Camden health systems, there is a potential for missing encounter data, although separate analyses by the Coalition have demonstrated that roughly $95 \%$ of hospital visits for Camden's adult population take place in Camden. However, the database also is vulnerable to movement in and out of the city, as nonresident data from the hospitals are not included.

Individuals in the Camden Health Database were identified as having diabetes through the diagnosis codes on the file (International Classification of Diseases, Ninth Revision codes used to identify persons with diabetes are 250, 357.2, 362.01-362.07, 366.41, V58.67). Individuals who were younger than age 18 years, who had diagnoses indicating type 1 diabetes or whose data were incomplete, were excluded from the linkage file. ED visits and inpatient stays are mutually exclusive events in the analyses. If a person was admitted to the hospital but had been admitted through the ED, the event was coded as an inpatient stay and did not count as an emergent visit. The study team calculated the hierarchical condition category score as a measure of health status for all individuals in the data set using records for 2 years before the date of record. The team also calculated a comorbidity index for all individuals in the data set using the Charlson comorbidity index. The final analytic data file constructed from the data set comprises records for 87,261 individuals.

\section{Methods}

The utilization analysis assessed 3 outcomes: (1) ED visit rates, (2) inpatient admission rates, and (3) total hospital charges. The study team analyzed these outcomes separately for the DSME participants. The analyses estimated intervention effects by assessing the changes in outcomes between the baseline (preintervention) period and the postintervention period. As part of this study, the study team also assessed changes in selected clinical values-hemoglobin A1c levels, blood pressure, weight, low-density lipoprotein (LDL) levels, and triglyceride levels-for DSME participants and for 1 comparison group.

\section{Design}

The study team completed 2 sets of utilization analyses on the DSME program (data set). One set of analyses used only the intervention data from the 123 participants to assess changes over time. The second set of analyses compared the outcomes from the participants with outcomes for 2 separate comparison groups. Comparison group 1 (CG-1) comprised 54 individuals who met the criteria for participation and who were on the recruitment list but who did not participate in the program. Comparison group 2 (CG-2) comprised individuals who were selected from the Camden Health Database, using propensity score methods to adjust CG-2 to resemble the in- tervention group more closely. Both stratification and covariate adjustment were used. Propensity scores were calculated using logistic regression with age, sex, and Charlson score as covariates to predict membership in the intervention group. Propensity score strata were then created by identifying the propensity score quantiles from the intervention group and then identifying individuals in CG-2 who had propensity scores within the 5 strata defined by these quantile values. Each propensity score stratum represents a group of comparison and intervention participants who have similar propensity scores - and thus similar characteristicsas defined by the propensity score quintiles. These propensity score strata were then also used in the analysis both as a stratifying variable and as a covariate.

The study team also calculated the mean changes in clinical measures for participants at the beginnings of their periods of participation and afterward. The team also calculated mean changes for CG-1 over the same periods. Clinical measures were available for a subset of participants and comparisons because they were taken during periodic medical visits and were completed by physicians as part of providing routine medical care.

\section{Study period}

A 3-year period was selected for assessing the outcomes: from 2010 through 2012. Up to 12 quarterly observations were used per participant. Some of the quarters were for periods before the start of the DSME program, and other quarters occurred after the start of the DSME program. The DSME course was repeated for different sets of enrollees. Start dates for participants varied according to the iteration in which they enrolled. The study team removed the quarter in which a participant started the intervention because it was not apparent whether that quarter should be considered a baseline observation or a postintervention observation. Some DSME participants had quarterly data only during the baseline period (before the Camden programs were taking place), whereas others contributed to both the baseline period and the postprogram period.

\section{Regression models}

Multivariate regression models were used for the utilization analyses. Generalized estimating equations (GEEs), Poisson, and nonlinear zero-inflated Poisson regression models were used to estimate changes in hospitalization rates and ED visit rates. Logistic gamma hurdle models were used to assess costs. Models estimated the changes in both utilization rates and costs over time for the participant groups, as well as changes over time relative to the changes over time for the comparison groups. The models used to compare the participant group with the comparison group included an indicator for the group (intervention or comparison) and were either $(a)$ stratified by the propensity score quintile group or (b) the propensity score quintile group was used as a 5-level factor covariate. The study team selected these models because, in most periods (in this case, quarters), most individuals will not use hospital services or have costs. The models assess both the likelihood of having any costs, visits, or stays and the amount of costs, visits, or stays given that individuals had nonzero numbers of these. The models controlled for payer type, sex, age, time, comorbidities, and whether the 
participation in the DSME was low intensity or high intensity. (The GEE models included payer type and sex as fixed covariates, while time, age, Charlson score, and intervention utilization were time-dependent covariates. The time covariate was a 12-level factor variable, 1 level for each quarter during 2010 through 2012. The payer type covariate was a 5 -level factor variable. The intervention intensity covariate was a 3-level factor variable: pre, low, and high.) The pre level represents quarters before the start of any intervention, the low level represents quarters in which the subject was identified as a low-intensity participant in the intervention, and the high level represents quarters in which the subject was identified as a high-intensity participant.

The results are estimated ratios of mean utilization rates and costs among periods of high-intensity participation relative to periods of preintervention participation. (The zero-inflated Poisson models included random intercepts for subjects in the logistic component, whereas the Poisson component included random subject intercepts, age, sex, Charlson score, payer type, and intervention levels as covariates. The logistic gamma hurdle models included random subject intercepts in the logistic component, and the gamma component included random subject intercepts, age, sex, Charlson score, payer type, and intervention levels as covariates.) Results from the models that included a comparison group were estimated ratios of mean utilization rates and costs among preintervention periods, lowintensity participation periods, and high-intensity participation periods relative to the comparison group.

\section{Results}

\section{Use of hospital services}

Table 1 presents the annual utilization rates and monthly costs for participants in the DSME program and for both comparison groups. The table presents the rates (for inpatient stays and ED visits) and costs (for charges) for program participants in the baseline (pre-program) period (beginning in 2010), followed by the rates and costs in the period after participation.
Baseline inpatient rates for participants were about 0.32 per person annually (equivalent to 1 stay every 3 years per person) and increased slightly after the program. ED visit rates were about one per year both before and after the program. ED visit rates for individuals in CG-1 were slightly higher; rates for CG-2 were about the same as the participants. Before the start of the program, hospital costs were close to $\$ 1600$ monthly for the participant group (Table 1). In the years after the DSME program began, costs were approximately $\$ 3000$ for both the participants and the persons in CG-1. Costs were lower for persons in CG-2.

The first analyses assessed the change in outcomes after participation in the DSME program (data not shown). Inpatient stays declined by about half among the low-intensity group (rate ratio of $0.45 ; P=0.02$ ) but did not change among the high-intensity participants. ED visit rates remained about the same over time for both groups. The high-intensity group incurred greater hospital charges after participation (ratio of $1.80 ; P=0.03$ ), and charges remained about the same for the low-intensity group.

There were few differences between low- and highintensity participants. In fact, low-intensity participants appeared to have lower hospital costs and fewer hospital admissions than the high-intensity group. This is counterintuitive and contradicts the study hypothesis that high-intensity participants would be more likely to have lower costs and utilization rates after graduating from the program.

The next set of analyses, summarized in Table 2, assessed the changes in outcomes relative to the 2 comparison groups: CG-1 being those who met the criteria for enrollment but did not participate and CG-2 being persons in the Camden Health Database who were assigned to a second comparison group using propensity score methods. Results are expressed as the ratio of events or costs for the participants relative to each comparison group in both the baseline and post-program periods.

Participants in the DSME program had slightly lower inpatient and ED visit rates over time relative to persons in the comparison groups. Relative to CG-1, inpatient rates were

Table 1. Utilization Rates for DSME Program Participants and Comparison Groups

\begin{tabular}{|c|c|c|c|c|c|}
\hline \multirow[b]{2}{*}{ Outcome measure } & \multirow{2}{*}{$\begin{array}{c}\text { Baseline rates } \\
\text { Low/high }(\mathrm{n}=123) \\
(P Y=271)\end{array}$} & \multicolumn{4}{|c|}{ Postprogram rates } \\
\hline & & $\begin{array}{c}\text { High intensity } \\
(\mathrm{n}=48)(P Y=30.25)\end{array}$ & $\begin{array}{c}\text { Low intensity } \\
(\mathrm{n}=50)(P Y=41.5)\end{array}$ & $\begin{array}{c}C G-1(\mathrm{n}=84) \\
(P Y=252)\end{array}$ & $\begin{array}{c}C G-2(\mathrm{n}=9006) \\
(P Y=27,018)\end{array}$ \\
\hline \multicolumn{6}{|c|}{ Inpatient stays (per year) } \\
\hline Total & 88 & 12 & 17 & 119 & 7659 \\
\hline Rate & 0.32 & 0.40 & 0.41 & 0.47 & 0.28 \\
\hline CI & $(0.25,0.40)$ & $(0.17,0.62)$ & $(0.22,0.60)$ & $(0.38,0.55)$ & $(0.28,0.29)$ \\
\hline \multicolumn{6}{|c|}{ Emergency department visits } \\
\hline Total & 290 & 29 & 42 & 346 & 23,765 \\
\hline Rate & 1.07 & 0.96 & 1.01 & 1.37 & 0.88 \\
\hline CI & $(0.95,1.19)$ & $(0.61,1.31)$ & $(0.71,1.32)$ & $(1.22,1.51)$ & $(0.86,0.89)$ \\
\hline \multicolumn{6}{|l|}{ Costs } \\
\hline$\$$ per month & 1579 & 3088 & 2993 & 2919 & 2071 \\
\hline $\mathrm{CI}$ & \pm 411 & \pm 2031 & \pm 2202 & \pm 750 & \pm 76 \\
\hline
\end{tabular}

CG-1 comprised 54 individuals who met the criteria for participation and who were on the recruitment list but who did not participate in the program. CG-2 comprised individuals selected from the Camden Citywide Claims data set, using propensity score methods to adjust CG-2 to resemble the intervention group more closely.

CI, 95\% confidence interval; CG-1, comparison group 1; CG-2, comparison group 2; DSME, diabetes self-management education; PY, person-years. 
Table 2. Rate Ratios Comparing the DSME Intervention Group and Comparison Groups

\begin{tabular}{|c|c|c|c|c|}
\hline \multirow[b]{2}{*}{ Outcome measure } & \multicolumn{2}{|c|}{$D S M E$ participants relative to $C G-1^{\mathrm{a}}$} & \multicolumn{2}{|c|}{ DSME participants relative to $C G-2^{\mathrm{b}}$} \\
\hline & Pre & Post & Pre & Post \\
\hline \multicolumn{5}{|c|}{ Inpatient stays (per year) } \\
\hline Rate ratio & $0.97(P=0.86)$ & $0.52(P=0.33)$ & $1.41(P=0.09)$ & $1.33(P=0.16)$ \\
\hline CI & $0.65,1.44$ & $0.14,1.92$ & $0.94,2.10$ & $0.90,1.97$ \\
\hline \multicolumn{5}{|c|}{ Emergency department visits } \\
\hline Rate ratio & $0.86(P=0.40)$ & $0.77(P=0.51)$ & $0.99(P=0.95)$ & $0.94(P=0.84)$ \\
\hline CI & $0.62,1.21$ & $0.35,1.67$ & $0.76,1.29$ & $0.54,1.64$ \\
\hline \multicolumn{5}{|l|}{ Costs } \\
\hline Cost ratio & $1.01(P=0.99)$ & $1.27(P=0.72)$ & - & - \\
\hline CI & $0.39,2.63$ & $0.33,4.92$ & - & - \\
\hline
\end{tabular}

CG-1 comprised 54 individuals who met the criteria for participation and who were on the recruitment list but who did not participate in the program. CG-2 comprised individuals selected from the Camden Citywide Claims data set, using propensity score methods to adjust CG-2 to resemble the intervention group more closely.

${ }^{a}$ Utilization measures were analyzed using a zero-inflated Poisson model. Costs were analyzed using a logistic gamma hurdle model. Statistics for post results are based on weighted averages of model results from low- and high-intensity participants.

${ }^{b}$ Utilization measures were analyzed using a GEE Poisson model, where propensity score quintile group was used as a covariate. Costs were not analyzed using GEE methodology because of non-normality. GEE models instead of zero-inflated/hurdle models were used in this analysis because of large sample size. Statistics for post results are based on weighted averages of model results from low- and highintensity participants.

CI, confidence interval; DSME, diabetes self-management education; GEE, generalized estimating equation.

about the same in the baseline period but were about half those of CG-1 after the program (Table 2). This relative decline was not statistically significant, however. ED visit rates were slightly lower than those in CG-1 in both periods, and the rate ratios changed little and were not statistically significant. Costs were essentially the same among participants and comparisons in the baseline period but were about $25 \%$ higher in the post period for the DSME participants relative to the comparisons. Relative to CG-2, inpatient rates were higher for DSME participants both before and after the program took place and also improved slightly in the post period. The relative difference was small and not statistically significant. As reported in the tables, the study did not find reductions in the use of hospital services or costs among participants in the DSME program over time or relative to the comparisons over time.

\section{Clinical measures}

Mean hemoglobin A1c levels for both the participants and nonparticipants improved by 3 percentage points, but the overall levels were much lower for participants: final values were 7.9 for participants and 8.8 for nonparticipants (data not shown). Both LDL levels and triglyceride levels improved for participants (by 6 and $8 \mathrm{mg} / \mathrm{dL}$, respectively) but remained essentially the same for nonparticipants. Changes in blood pressure and weight loss could not be assessed for participants because only 15 participants were measured for these. Changes in clinical indicators associated with the DSME program were promising but were not statistically significant because of small sample sizes. Results from the 5 sites combined, which were part of the Alliance to Reduce Disparities in Diabetes, showed statistically significant decreases in hemoglobin A1c levels and blood pressure. ${ }^{13}$

\section{Discussion}

Funders, stakeholders, and policy makers often expect programs for persons with chronic conditions such as dia- betes to "bend the cost curve" for the individuals served, primarily by reducing the use of expensive hospital services. This study assessed whether a diabetes self-management program, as part of a multicomponent, multilevel initiative, improved costs in the short run, specifically as related to hospital services such as ED visits and inpatient stays.

This study found no bend in the cost curve for those participating in the DSME programs as had been previously found for some care management interventions for high health care utilizers. ${ }^{9}$ DSME deployed at the community level, without explicit segmentation and targeting of high health care utilizers, might not reduce short-term medical needs for participating individuals. Many medical needs and reasons for inpatient stays and ED visits are not directly related to diabetes (although any chronic illness may affect symptoms caused by other illnesses or how a patient is treated for other conditions). These individuals might continue to have hospitalizations or ED visits that are not closely related to or caused by having diabetes, and thus, they may not have been affected by the DSME program. High utilization rates and costs for diabetes tend to be associated with longer term progression of the disease and its sequelae; thus, DSME or peer support may not affect these values over the course of a 1- to 3-year study but could have an affect later in individuals' lives.

There are several possibilities to consider regarding the observed outcomes for participants in the DSME program. The sample size for this study was small, with 123 persons participating in the DMSE program and 48 completing it, and with 98 having post-period observations. Consequently, results related to short-term use of hospital services and the associated costs could not be detected. One limitation of this study is the likely nonequivalence of the comparison groups and participant groups. The baseline hemoglobin A1c values for nonparticipants in CG-1 were almost a full percentage point higher than for the comparison population, suggesting that the 2 groups were different in the progression of their 
diabetes when the DSME program began. This discrepancy likely is correlated with the willingness to participate in the program.

The goal of DSME is to slow disease progression so a portion of the long-term adverse events associated with diabetes can be avoided, resulting in lower utilization and costs. Adverse events related to diabetes include kidney failure, amputations, and diabetic neuropathy. These sequelae are not frequent events among persons with recent diagnoses. This study did not assess the longer term effects of DSME; rather, it focused solely on near-term effects. The observed improvements in some clinical measures could affect costs and utilization in the longer term, but expecting educational programs to lead to short-term cost savings is not realistic.

The baseline utilization rates of about 0.3 annually for inpatient stays and about 1 annually for emergent visits for DSME participants, while higher than average, were not at the levels seen for individuals who would be considered high utilizers of hospital services in the Camden area. Even if the hospital use was considered excessive, neither the goals nor the curriculum of the DSME program was intended to address use of hospital services directly. The DSME program addresses how to achieve control of clinical indicators, how to live a healthier life with diabetes, and how to manage acute and chronic symptoms associated with diabetes. A different kind of intervention with an explicit strategy to reduce overuse of hospital services would be needed to address what would be considered routinely unnecessary use for this group of participants or others. The results of this study can inform expectations about interventions for underserved populations and evaluation design features needed to discern and interpret program effects, as well as expectations related to cost savings. If affecting utilization and costs are a program's objective, communities, policy makers, and stakeholders need to work with payers, patients, and providers to ensure programs have specific components in place to affect them. Successful population health programs recognize the need to include process, clinical, service, and financial outcomes in the design of their programs.

\section{Acknowledgments}

The authors thank Shawn Karns at RTI International for expert programming support, Connie Hobbs at RTI who provided administrative support, and Loretta Bohn at RTI who provided editorial assistance to the authors.

\section{Author Disclosure Statement}

Mr. Burton, Mr. Eggleston, Dr. Brenner, Mr. Truchil, Dr. Lewis, and Ms. Zulkiewicz declared no conflicts of interest. The authors received the following financial support. This manuscript was prepared and the analyses described completed with funding from the Merck Company Foundation as part of its Alliance to Reduce Disparities in Diabetes. The Camden Coalition of Healthcare Providers was one of the Alliance-funded grantees, and RTI International received a contract to evaluate the Alliance-funded programs. The contents of the manuscript were derived solely by the authors, none of whom had a conflict of interest in submitting this manuscript other than any perceptions stemming from the receipt of funds from the Merck Company Foundation.

\section{References}

1. American Diabetes Association. Fast facts: data and statistics about diabetes. 2015. http://professional2.diabetes. org/admin/UserFiles/0\%20-\%20Sean/Documents/Fast_Facts_ 12-2015a.pdf Accessed March 24, 2016.

2. Centers for Disease Control and Prevention. National Diabetes Statistics Report, 2014. www.cdc.gov/diabetes/pdfs/ data/2014-report-estimates-of-diabetes-and-its-burden-in-theunited-states.pdf Accessed March 24, 2016.

3. Agency for Healthcare Research and Quality. National Healthcare Disparities Report, 2008. AHRQ Publication No. 09-0002. Rockville, MD: US Department of Health and Human Services, Agency for Healthcare Research and Quality, 2009. AHRQ Publication No. 09-0002.

4. Huang ES, Basu A, O'Grady M, Capretta JC. Projecting the future diabetes population size and related costs for the U.S. Diabetes Care 2009;32:2225-2229.

5. Boyle JP, Honeycutt AA, Narayan KM, et al. Projection of diabetes burden through 2050: impact of changing demography and disease prevalence in the U.S. Diabetes Care 2001;24:1936-1940.

6. Gross K, Brenner JC, Truchil A, Post EM, RIley AH. Building a citywide, all-payer, hospital claims database to improve health care delivery in a low-income, urban community. Popul Health Manag 2013;16 (suppl 1):S20S25.

7. Gawande A. The hot spotters: can we lower medical costs by giving the neediest patients better care? www.newyorker .com/magazine/2011/01/24/the-hot-spotters Accessed March 23, 2016.

8. Clark NM, Brenner J, Johnson P, et al. Reducing disparities in diabetes: the alliance model for health care improvements. Diabetes Spectr 2011;24:226-230.

9. Kaufman S, Ali N, DeFiglio V, Craig K, Brenner J. Early efforts to target and enroll high-risk diabetic patients into urban community-based programs. Health Promot Pract 2014;15 (2 Suppl):62s-70s.

10. Green SR, Singh V, O'Byrne W. Hope for New Jersey's city hospitals: the Camden initiative. Perspect Health Inf Manag 2010;7:1d.

11. Coburn KD, Marcantonio S, Lazansky R, Keller M, Davis $\mathrm{N}$. Effect of a community-based nursing intervention on mortality in chronically ill older adults: a randomized controlled trial. PLoS Med 2012;9:e1001265.

12. Philis-Tsimikas A, Fortmann A, Lleva-Ocana L, Walker C, Gallo LC. Peer-led diabetes education programs in highrisk Mexican Americans improve glycemic control compared with standard approaches: a Project Dulce promotora randomized trial. Diabetes care 2011;34:1926-1931.

13. Lewis MA, Bann CM, Karns SA, et al. Cross-site evaluation of the alliance to reduce disparities in diabetes: clinical and patient-reported outcomes. Health Promot Pract 2014; 15 (2 suppl):92S-102S.

Address correspondence to: Joe Burton, $M S$ RTI International 307 Waverley Oaks Road, Suite 101 Waltham, MA 02452-8413

E-mail: joeburton@rti.org 